\title{
Optimal Braking Force Allocation for a Four Wheel Drive Fully Electric Vehicle
}

\author{
A. Pennycott*, L. De Novellis, P. Gruber and A. Sorniotti \\ Department of Mechanical Engineering Sciences \\ University of Surrey \\ United Kingdom
}

\section{Abstract}

Control allocation can be used onboard fully electric vehicles in order to maximise the regenerative power produced during braking manoeuvres. In this study, the efficiency characteristics of an electric motor are used in conjunction with constraints from European braking regulations in an offline optimisation procedure aimed at maximising the regenerative power yielded at different motor speed and braking demand conditions. The resulting optimisation data are used in a simple online control allocation approach via a look-up table. Simulation results highlight significant motor power loss reductions and small increases in regenerative power under various levels of braking demand in comparison with a wheel torque allocation scheme in which the front axle to total braking force ratio is maintained at a constant level. The approach does not rely on complex online optimisation schemes and can thus be practically implemented in real time on fully electric vehicles.

Keywords: fully electric vehicles; control allocation; energy efficiency

\section{Nomenclature}

\section{Symbols}

$\gamma \quad$ Ratio of front axle to total braking force

$\kappa \quad$ Transmission ratio

$\omega_{m} \quad$ Motor speed $[\mathrm{rad} / \mathrm{s}]$

$\tau_{m} \quad$ Motor torque $[\mathrm{Nm}]$

$\tau_{w}^{\text {tot }} \quad$ Total wheel torque demand $[\mathrm{Nm}]$

$a_{x} \quad$ Longitudinal acceleration $\left[\mathrm{m} / \mathrm{s}^{2}\right]$

$a_{x}^{\text {err }} \quad$ Longitudinal acceleration error $\left[\mathrm{m} / \mathrm{s}^{2}\right]$

$a_{x}^{\text {ref }} \quad$ Reference longitudinal acceleration $\left[\mathrm{m} / \mathrm{s}^{2}\right]$

*a.pennycott@surrey.ac.uk 


$\begin{array}{ll}b_{f} & \text { Front semi-wheelbase }[\mathrm{m}] \\ b_{r} & \text { Rear semi-wheelbase }[\mathrm{m}] \\ F_{x}^{\text {tot }} & \text { Braking force demand }[\mathrm{N}] \\ h & \text { Height of centre of mass }[\mathrm{m}] \\ k_{f} & \text { Front adhesion utilisation coefficient } \\ k_{r} & \text { Rear adhesion utilisation coefficient } \\ L & \text { Wheelbase }[\mathrm{m}] \\ m & \text { Mass [kg] } \\ M_{z} & \text { Yaw moment [Nm] } \\ P & \text { Power [kW] } \\ P_{\text {loss }} & \text { Power loss }[\mathrm{kW}] \\ P_{\text {regen }} & \text { Regenerative power }[\mathrm{kW}] \\ r_{w} & \text { Wheel radius }[\mathrm{m}] \\ t_{h} & \text { Half-track }[\mathrm{m}] \\ z & \text { Braking intensity }\end{array}$

\section{Introduction}

There is growing interest in electric vehicles (EV) due to a number of factors including a drive towards energy diversification in the transport sector [1] and concerns regarding the cost and longterm availability of fossil fuels [2, 3]. Furthermore, EVs have benefits with regard to emissions output [4], which is of particular importance in light of the possible contribution of transportation to anthropomorphic climate change [5]. In spite of their various benefits, battery life and vehicle range remain major drawbacks of EVs $[6,7,8]$. However, recent developments in braking technology enable continuous modulation of braking [9]; moreover, the braking torques applied to the individual wheels can be accurately and independently controlled [10]. As a result, methods based on torque vectoring aimed at increasing energy efficiency could help reduce the severity of these limitations.

For braking manoeuvres, the electric motors of the car can be applied as generators, with the generated electricity being used to charge the battery. This energy recovery mechanism can yield appreciable power savings during vehicle operation [11]. The inclusion of four individually-controlled electric motors allows the regenerative braking force demand to be met via an infinite number of combinations in the individual wheel torques. For instance, a target braking force can be realised by assigning the entire demand to the wheels of the front axle, the rear axle, or equally distributed between the front and rear axles. Due to the variation in motor efficiency throughout the speedtorque space, the various combinations will yield different levels of power loss and therefore power available for charging the battery. If data concerning the motor characteristics are available, control allocation - the process of assigning control signals to the individual actuators in order to realise an overall target such as the net braking force [12] - allows the wheel torque combination to be determined such that the total regenerative power is maximised. 
Indeed, various studies have investigated the potential of control allocation to deliver improvements in energy efficiency for different types of motor $[13,14]$. However, these have tended to rely on the minimisation of a polynomial cost function online, a task which may be difficult to perform in real time applications due to the computational demands of the optimisation scheme. Moreover, during a braking manoeuvre, additional constraints must be taken into account which arise from braking regulations aimed at preventing wheel lock of the rear axle, requiring further modification of optimisation-based wheel torque allocation schemes.

In this paper, a control allocation approach is developed for a fully electric vehicle (but could also be adapted for hybrid vehicles) with four individually controlled drivetrains. The method incorporates both the efficiency characteristics of the electric motor actuators and the braking regulation constraints within an offline optimisation procedure. The data generated from the optimisation procedure are included for online wheel torque allocation in the form of a look-up table, an approach which allows simple implementation on actual vehicles. Using a simulation approach, the regenerative power produced under this scheme is compared to a simpler control allocation approach in which the front-to-total braking force ratio is maintained at a constant level.

\section{Methods}

\subsection{Vehicle Geometry}

The front and rear semi-wheelbases, $b_{f}$ and $b_{r}$, and the height of the centre of mass of the car above the ground, $h$, are key parameters for braking manoeuvres. These geometric parameters are illustrated in figure 1. Throughout the derivations of this paper, the wheels of the front-left, front-right, rear-left and rear-right are given the subscripts 1, 2, 3 and 4, respectively.

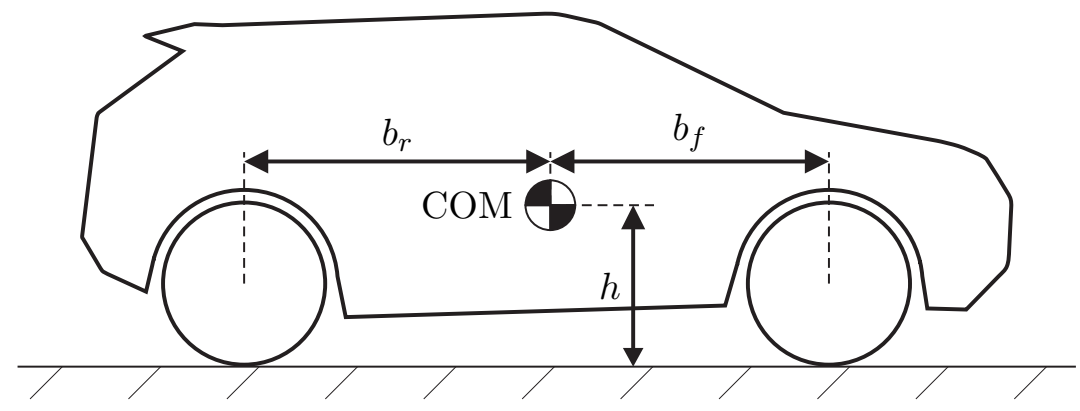

Figure 1: Vehicle geometry.

The vehicle simulated in this study has a front semi-wheel base $\left(b_{f}\right)$ of $1.07 \mathrm{~m}$, a rear semi-wheelbase $\left(b_{r}\right)$ of $1.59 \mathrm{~m}$ and the distance from the ground to the centre of gravity $(h)$ is $0.66 \mathrm{~m}$.

\subsection{Braking Constraints}

If the rear wheels lock during braking, instability may result due to vehicle oversteer. In order to avoid this situation, a number of conditions are provided in Regulation 13 of the Economic Commission for Europe (ECE) [15], in which constraints are specified concerning the ratio of the front axle braking force to the total braking force, $\gamma[16] . \gamma$ is thus defined as

$$
\gamma=\frac{F_{x, f}}{F_{x}^{\text {tot }}}
$$

where $F_{x}^{\text {tot }}$ is the total braking force demand and $m$ is the mass of the car. 
In order to produce a given longitudinal acceleration, $a_{x}$, the required total longitudinal force is $F_{x}^{\text {tot }}=m a_{x}$, hence the required longitudinal forces developed at the front and rear axles are

$$
\begin{aligned}
& F_{x, f}=m a_{x} \gamma \\
& F_{x, r}=m a_{x}(1-\gamma) .
\end{aligned}
$$

The vertical loads on the front and rear axles $\left(F_{z, f}\right.$ and $\left.F_{z, r}\right)$ are

$$
\begin{aligned}
& F_{z, f}=\frac{m g b_{r}}{L}-\frac{m h a_{x}}{L} \\
& F_{z, r}=\frac{m g b_{f}}{L}+\frac{m h a_{x}}{L}
\end{aligned}
$$

in which $L$ is the wheelbase (equal to $b_{f}+b_{r}$ ).

The braking intensity, $z$, is defined as

$$
z=-\frac{F_{x}^{\mathrm{tot}}}{m g}=-\frac{a_{x}}{g}
$$

The adhesion utilisation $k$, defined as the ratio of the braking force to the maximum braking force at a given axle, can be determined in terms of the braking intensity. In the following derivations, the friction coefficient is set to 1 ; however, the equations can be altered appropriately for different values of friction coefficient arising from different surface conditions. For the front and rear axles, the adhesion utilisations are

$$
\begin{aligned}
& k_{f}=-\frac{m a_{x} \gamma}{\left(m g b-m h a_{x}\right) / L}=\frac{\gamma z L}{b_{r}+z h} \\
& k_{r}=-\frac{m a_{x}(1-\gamma)}{\left(m g b_{f}+m h a_{x}\right) / L}=\frac{(1-\gamma) z L}{a-z h} .
\end{aligned}
$$

Requirement 3.1.1 of the braking regulations stipulates that for an adhesion utilisation coefficient between 0.2 and 0.8 , the following inequality is applicable for braking intensities between 0.1 and 0.61:

$$
z \geq 0.1+0.85(k-0.2)
$$

which leads to

$$
0.85 k \leq z+0.07
$$

Secondly, requirement 3.1.2 is that the friction utilisation of the front axle should be greater or equal to that of the rear axle.

$$
k_{f} \geq k_{r}
$$

Using the definitions of the front and rear utilisation coefficients of equations (7) and (8), inequality (11) yields

$$
\begin{gathered}
\frac{\gamma z L}{b_{r}+z h} \geq \frac{(1-\gamma) z L}{b_{f}-z h} \\
\Leftrightarrow \gamma\left(b_{f}+b_{r}\right) \geq b_{r}+z h
\end{gathered}
$$

and therefore, since $L=b_{f}+b_{r}$, for all braking conditions:

$$
\gamma \geq \frac{b_{r}+z h}{L}
$$


Concerning the front axle, at braking intensities between 0.1 and 0.61 , inequality (10) yields

$$
\begin{gathered}
\frac{0.85 \gamma z L}{b_{r}+z h} \leq z+0.07 \\
\Leftrightarrow \gamma \leq \frac{(z+0.07)\left(b_{r}+z h\right)}{0.85 z L}
\end{gathered}
$$

and similarly, for the rear axle:

$$
\begin{gathered}
\frac{0.85(1-\gamma) z L}{b_{f}-z h} \leq z+0.07 \\
\Leftrightarrow \gamma \geq 1-\frac{(z+0.07)\left(b_{f}-z h\right)}{0.85 z L} .
\end{gathered}
$$

Using the geometry of the vehicle (section 3.1), the permitted values of $\gamma$ at different levels of braking intensity are shown in figure 2. It can be seen that in the case of the specific vehicle in this paper, the constraints corresponding to the braking regulations can be completely described by inequalities (12) and (13). Furthermore, for lower levels of braking intensity, the upper and lower limits for $\gamma$ are insensitive to changes in $h$ (the height of the centre of gravity). For instance, at a braking intensity of 0.15 , changes in $h$ of $10 \%$ alter the limits by under $1 \%$ for the vehicle studied in this paper.

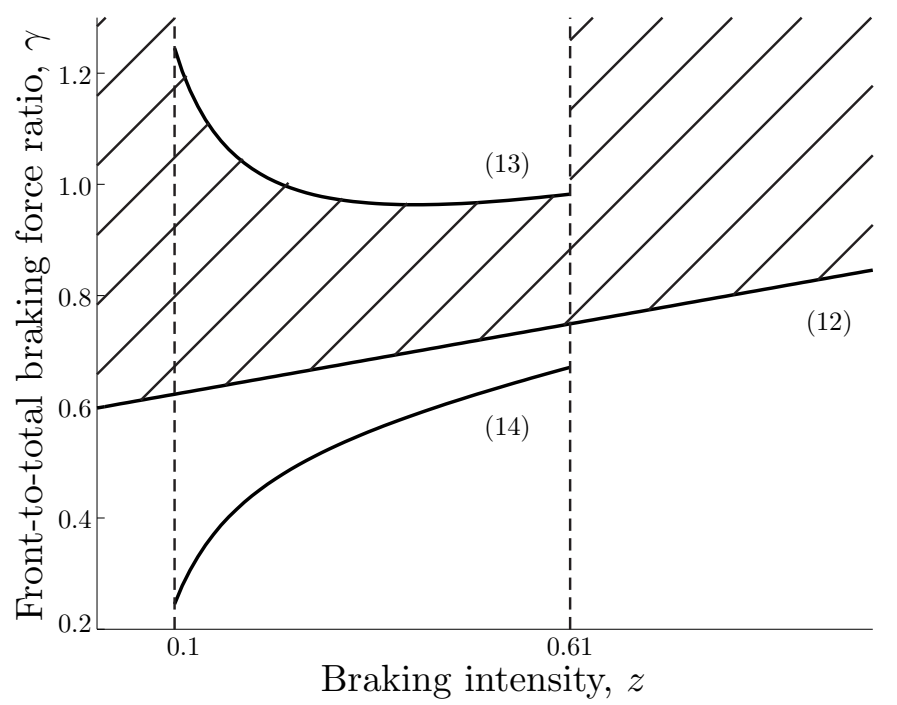

Figure 2: Constraints from the ECE braking regulations concerning the front-to-total braking force ratio, $\gamma$, with shaded areas corresponding to permitted values of the ratio.

\subsection{Energy Efficient Braking Allocation}

\subsubsection{Control Allocation and Power Derivations}

The control allocation equations relate the net longitudinal force, $F_{x}^{\text {tot }}$, and the net yaw moment, $M_{z}$, with the four wheel torques, $\tau_{w, 1-4}$, via the vehicle parameters of the wheel radius, $r_{w}$, and the half-track, $t_{h}$. The net longitudinal force is

$$
F_{x}^{\text {tot }}=\frac{1}{r_{w}}\left(\tau_{w, 1}+\tau_{w, 2}+\tau_{w, 3}+\tau_{w, 4}\right) .
$$


Using the half track, $t_{h}$, which is equal for the front and rear axles, the net yaw moment induced by the wheel torques, $M_{z}$, is equal to

$$
M_{z}=-t_{h} \tau_{w, 1}+t_{h} \tau_{w, 2}-t_{h} \tau_{w, 3}+t_{h} \tau_{w, 4}
$$

and since $M_{z}=0$ for the manoeuvre presented in this paper:

$$
-\tau_{w, 1}+\tau_{w, 2}-\tau_{w, 3}+\tau_{w, 4}=0 .
$$

Let the total wheel torque be $\tau_{w}^{\text {tot }}$, i.e.

$$
\tau_{w}^{\mathrm{tot}}=\sum_{i=1}^{4} \tau_{w, i} .
$$

The solution for the four wheel torques can be expressed in terms of two independent variables, $\Delta \tau_{l}$ and $\Delta \tau_{r}$, which represent the deviations of the allocated wheel torques from the solution corresponding to even braking distribution across the four wheels.

$$
\begin{aligned}
& \tau_{w, 1}=\tau_{w}^{\text {tot }} / 4-\Delta \tau_{l} \\
& \tau_{w, 2}=\tau_{w}^{\text {tot }} / 4-\Delta \tau_{r} \\
& \tau_{w, 3}=\tau_{w}^{\text {tot }} / 4+\Delta \tau_{l} \\
& \tau_{w, 4}=\tau_{w}^{\text {tot }} / 4+\Delta \tau_{r}
\end{aligned}
$$

The total wheel torques generated on the left- and right-hand sides of the vehicle are thus constant, with both being equal to $\tau_{w}^{\text {tot }} / 2$. The wheel torque allocation problem comprises the selection of $\Delta \tau_{l}$ and $\Delta \tau_{l}$ such the total regenerative power is maximised.

The efficiency of the motor units depends primarily on the motor speed, $\omega_{m}$, and motor torque, $\tau_{m}$. In steady state, the motor torque and wheel torque at wheel $i$ are approximately related via the equation $\tau_{w, i}=\kappa \tau_{m, i}$, where $\kappa$ is the transmission ratio. In straight-ahead driving and for a fixed transmission ratio, the motor speeds at each of the four actuators are roughly equal. For the left-hand side of the vehicle, there exists a value of $\Delta \tau_{l}$ that maximises the total regenerative power from the front-left and rear-left actuators. Since the motor speeds and total wheel torques at the two sides of the car are equal, the wheel torque assignment problem at the left- and right-hand sides of the car is symmetric, i.e. the value of $\Delta \tau_{l}$ that minimises the left-hand motor loss is equal to that of $\Delta \tau_{r}$ for the right-hand motors. Therefore, to maximise the regenerative power for a straight-ahead braking manoeuvre:

$$
\Delta \tau_{l}=\Delta \tau_{r}
$$

and therefore

$$
\begin{aligned}
& \tau_{w, 1}=\tau_{w, 2} \\
& \tau_{w, 3}=\tau_{w, 4} .
\end{aligned}
$$

There is thus only one degree of freedom to be determined by the wheel torque allocation scheme. The four wheel torques can be expressed via the ratio between the total wheel torque from the front axle to the total wheel torque. Since in steady state, the braking force at wheel $i, F_{x, i}$, is related to the wheel torque, $\tau_{w, i}$, via the equation $\tau_{w, i}=r_{w} F_{x, i}$ (where $r_{w}$ is the wheel radius, which is equal across all four wheels), the ratio between the front axle wheel torque to the total wheel torque is equal to the front-to-total braking force ratio, $\gamma$, defined in equation (1), i.e.

$$
\gamma=\frac{\tau_{w, 1}+\tau_{w, 2}}{\tau_{w}^{\text {tot }}}
$$


Using equations (23), (24) and (25), the individual wheel torques are then given via $\gamma$ :

$$
\begin{aligned}
\tau_{w, 1 / 2} & =\gamma \tau_{w}^{\mathrm{tot}} / 2 \\
\tau_{w, 3 / 4} & =(1-\gamma) \tau_{w}^{\mathrm{tot}} / 2
\end{aligned}
$$

For each motor $i$, during regenerative braking, the power delivered to the battery, $P_{\text {regen }, i}$ is

$$
P_{\text {regen }, i}=-P_{i} \eta\left(\omega_{m, i}, \tau_{m, i}\right)
$$

where $\eta\left(\omega_{m, i}, \tau_{m, i}\right)$ is the efficiency of the motor at motor speed $\omega_{m, i}$ and torque $\tau_{m, i}$ and the power, $P_{i}$, is simply the product $\tau_{m, i} \omega_{m, i}$. The efficiency is evaluated at different motor speed and torque conditions using an efficiency map provided by the manufacturer. The efficiency contours of the studied motor are depicted in figure 3 .

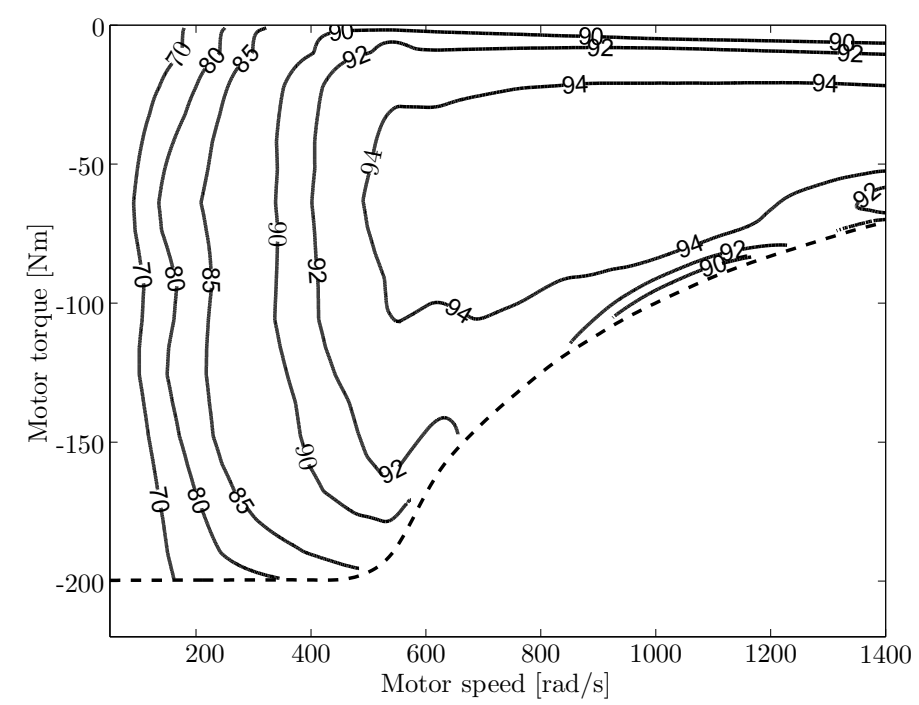

Figure 3: Efficiency contours (\%) of the motor at different levels of torque and speed. The dashed line indicates the maximum level of regenerative torque achievable at different motor speeds.

Since the wheel torques and hence the motor torques can be expressed as a function of $\tau_{w}^{\text {tot }}$ and $\gamma$, and also because the motor speeds are approximately equal at each wheel, the regenerative power (after losses from the actuators) can be written as a function of $\omega_{m}, \tau_{w}^{\text {tot }}$ and $\gamma$.

$$
\begin{aligned}
& P_{\text {regen }, 1 / 2}=-\frac{1}{2 \kappa} \omega_{m} \gamma \tau_{w}^{\mathrm{tot}} \eta\left(\omega_{m}, \frac{1}{2 \kappa} \gamma \tau_{w}^{\mathrm{tot}}\right) \\
& P_{\text {regen }, 3 / 4}=-\frac{1}{2 \kappa} \omega_{m}(1-\gamma) \tau_{w}^{\mathrm{tot}} \eta\left(\omega_{m}, \frac{1}{2 \kappa}(1-\gamma) \tau_{w}^{\mathrm{tot}}\right)
\end{aligned}
$$

The above expressions allow the total power available for charging the battery, $P_{\text {regen }}^{\text {tot }}$, to be written as a function in terms of $\gamma, \omega_{m}$ and $\tau_{w}^{\text {tot }}$.

$$
P_{\text {regen }}^{\text {tot }}=f\left(\gamma, \omega_{m}, \tau_{w}^{\text {tot }}\right)
$$

\subsubsection{Offline Optimisation}

An offline procedure is used to determine $\gamma^{*}$, the front-to-total braking force ratio that yields maximum regenerative power, throughout the speed-torque space. Using the total regenerative power 
function, $f$, of equation (32), at a given motor speed and torque demand, the optimal front-to-total ratio, $\gamma^{*}$, is calculated as

$$
\gamma^{*}=\underset{\gamma}{\arg \min }\left\{-f\left(\gamma, \omega_{m}, \tau_{w}^{\mathrm{tot}}\right)\right\}
$$

In addition to the cost function, the constraints from the ECE regulation detailed in section 3.2 must be taken into account. For each level of the braking torque target, $\tau_{w}^{\text {tot }}$, the braking intensity, $z$, is calculated according to equation (6), allowing the constraints of inequalities (12) and (13) to be generated. Let the minimum and maximum ratios given by the regulation be $\gamma_{\mathrm{ECE}}^{\min }$ and $\gamma_{\mathrm{ECE}}^{\max }$. The following constraints for $\gamma$ are used for the offline optimisation:

$$
\max \left(0, \gamma_{\mathrm{ECE}}^{\min }\right) \leq \gamma \leq \min \left(1, \gamma_{\mathrm{ECE}}^{\max }\right) .
$$

Furthermore, at each motor speed, $\omega_{m}$, there is a limiting value of torque that can be produced by the motors, $\tau_{m}^{\min }$. This gives further constraints on the front-to-total ratio of the form

$$
1-\frac{2 \kappa \tau_{m}^{\min }}{\tau_{w}^{\text {tot }}} \leq \gamma \leq \frac{2 \kappa \tau_{m}^{\min }}{\tau_{w}^{\text {tot }}}
$$

The optimisation problem is solved using the interior point method with the MATLAB command fmincon. Various starting points for the optimisation routine are included, with each generating a local maximum regenerative power (the total across the four actuators). The local solution with the highest regenerative power is then taken as the global solution.

A grid of motor speed and total wheel torque demand data is generated. Ranges of 0 - 1400 $\mathrm{rad} / \mathrm{s}$ and $-2000-0 \mathrm{Nm}$ are used for the motor speed and wheel torque demand, respectively, with $\gamma^{*}$ being calculated at each grid point. At a given point in the speed-torque space, the optimal front-to-total wheel torque ratio depends on the motor efficiency map, the motor torque limits and the restrictions imposed by the braking regulations.

\subsubsection{Online Wheel Torque Allocation}

The overall control scheme is shown in figure 4 . Based on the error between the reference $\left(a_{x}^{\text {ref }}\right)$ and actual $\left(a_{x}\right)$ longitudinal accelerations, $a_{x}^{\text {err }}$, the driver sets the total braking force demand, $F_{x}^{\text {tot }}$. In this research, the driver is modelled as a simple proportional controller with gain $K_{p}$. The braking force demand is converted to a total wheel torque demand, $\tau_{w}^{\text {tot }} \cdot \gamma^{*}$ is then determined from the motor speed and torque demand conditions via a 2-dimensional look-up table with linear interpolation; the resulting ratio is passed through a low pass filter (with cut-off frequency $1 \mathrm{~Hz}$ ) in order to avoid sharp changes in the motor torques which may cause drivability issues. The wheel torques are then realised by the electric motors ${ }^{1}$.

\subsection{Braking with Fixed Front-to-Total Ratio}

The energy efficient scheme detailed in section 3.3 is compared with a more basic control allocation approach in which the front-to-total braking force ratio is maintained at a constant level. The maximum deceleration tested in this study is $-2 \mathrm{~m} / \mathrm{s}^{2}$, giving a maximum braking intensity of 0.2 . At this braking intensity, the minimum permissible level of $\gamma$ according to the braking regulations is 0.65. A constant value for $\gamma$ corresponding to this value is therefore used for the fixed ratio allocation scheme so that the braking regulations are satisfied across all the simulated manoeuvres.

\footnotetext{
${ }^{1}$ For cases in which the motor torque demand exceeds the regenerative torque limits of the motor, the excess braking demand is realised from hydraulic braking.
} 


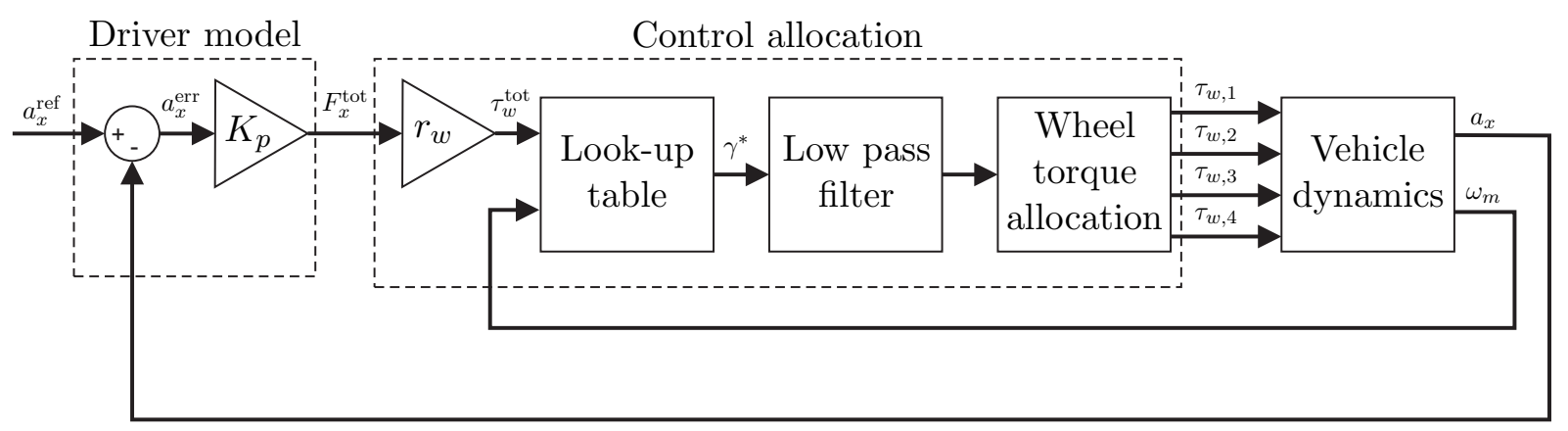

Figure 4: Schematic for online wheel torque allocation method.

\subsection{Simulation Study}

The ability of the two control allocation schemes with regard to delivering the required longitudinal acceleration and the total regenerative power achieved are compared using simulations of a model of a large electric vehicle developed in IPG CarMaker. In addition to the basic vehicle mechanics, the model includes the dynamics of the electric powertrains. The vehicle has a mass of $1963 \mathrm{~kg}$ and a fixed transmission ratio of 10:1.

Target longitudinal decelerations between -0.5 and $-2 \mathrm{~m} / \mathrm{s}^{2}$ are simulated. For each simulation run, the energy efficient and fixed front-to-total ratio control allocation schemes are compared in terms of the mean power loss, the mean regenerative power and the mean longitudinal acceleration. In each simulation, the manoeuvre comprises decelerating from 50 to $20 \mathrm{~km} / \mathrm{h}$. The conditions of the manoeuvre were chosen so as to reflect the region of the motor operating space in which differences between the two control allocation schemes compared in the paper would be expected (figure 6).

\section{Results}

\subsection{Offline Optimisation}

Figure 5 shows, for different levels of motor speed and total wheel torque demand, the values of the front-to-total braking force ratio at which the total regenerative power is minimised. Within certain areas of the torque-speed space, the braking force can be realised most efficiently by allocating almost the entire demand to the wheels of the front axle. Outside of this region at higher levels of braking demand and motor speed, however, the optimum ratio tends to fall to the minimum level as specified by the ECE regulation. Note that for all the simulations, the entire braking demand is met by the motor units alone, with no action being required from the hydraulic braking system. In the bottom-right region of the plot in figure 5, the optimal values of the front-to-total wheel torque ratio assume the lower limits of the ECE braking regulations (around 0.7).

Figure 6 shows the percentage increase in the total regenerative power delivered by the optimum braking allocation scheme relative to that produced with a fixed ratio of 0.65 . The optimum braking distribution method provides an increase in regenerative power in a narrow region of the torque-speed space; however, at higher wheel speeds, little benefit of the scheme is apparent for this particular motor type.

\subsection{Online Control Allocation}

In table 1 , the mean longitudinal acceleration is provided along with the mean (total) motor power loss incurred and mean regenerative power delivered during the various target acceleration conditions 


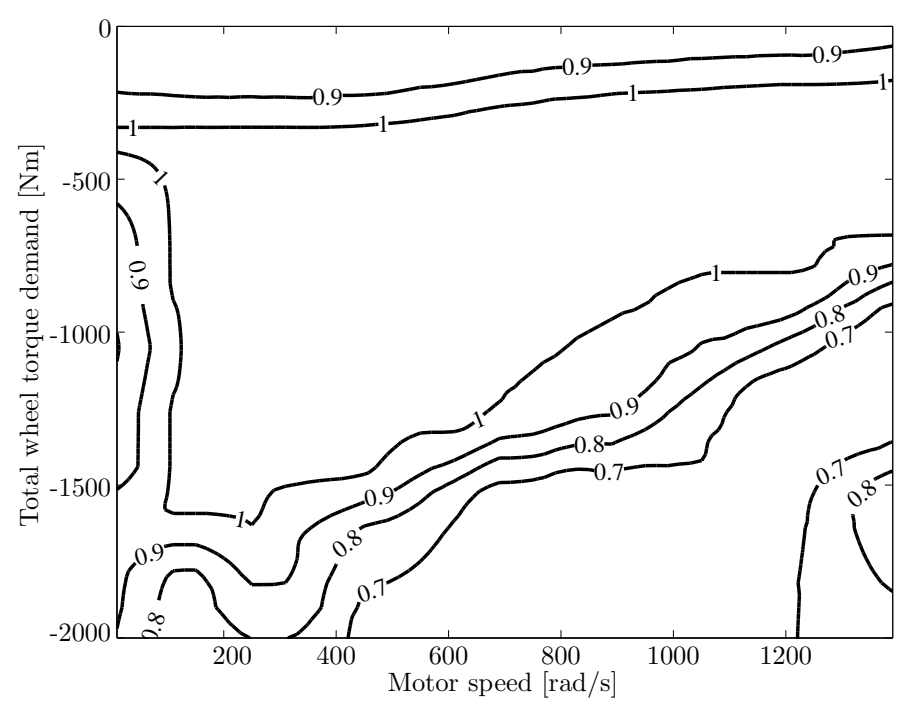

Figure 5: Optimal front-to-total braking force ratio contours throughout the torque-speed space.

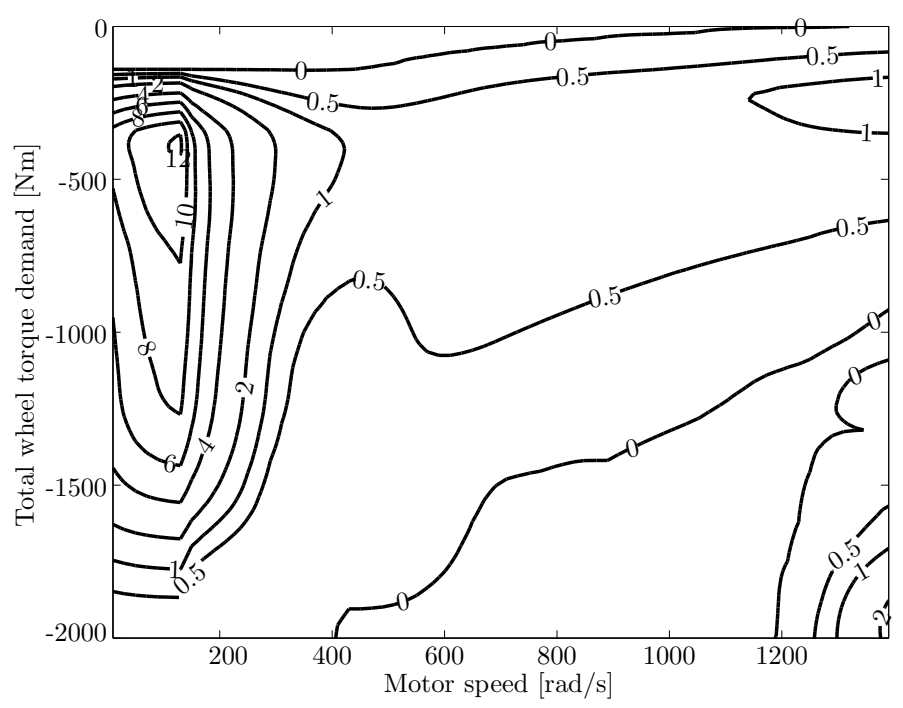

Figure 6: Contour plot showing the increase (\%) in regenerative power achieved by optimal braking allocation scheme relative to the fixed ratio method.

under the fixed ratio and energy efficient control allocation schemes. Both schemes satisfactorily realise the target longitudinal acceleration for all the manoeuvres. By varying the front-to-total braking force ratio according to the total braking demand and motor speed conditions, the energy efficient scheme achieves significant reductions in motor power loss as compared to the allocation scheme in which the front-to-total braking force (and therefore torque) ratio is fixed at $65 \%$. Power loss reductions of up to around $13 \%$ are achieved using the efficient control allocation approach. By operating in more efficient regions of the torque-speed space, the optimal braking distribution scheme produces small regenerative power increases relative to the fixed distribution method of over $2 \%$ in some conditions. 


\begin{tabular}{|c|c|c|c|c|c|c|}
\hline \multirow{2}{*}{$a_{x}^{\text {ref }}\left(\mathrm{m} / \mathrm{s}^{2}\right)$} & \multicolumn{3}{|c|}{ Fixed ratio allocation } & \multicolumn{3}{c|}{ Optimal braking } \\
\cline { 2 - 7 } & $a_{x}\left(\mathrm{~m} / \mathrm{s}^{2}\right)$ & $P_{\text {loss }}(\mathrm{kW})$ & $P_{\text {regen }}(\mathrm{kW})$ & $a_{x}\left(\mathrm{~m} / \mathrm{s}^{2}\right)$ & $P_{\text {loss }}(\mathrm{kW})$ & $P_{\text {regen }}(\mathrm{kW})$ \\
\hline-0.50 & -0.499 & 1.32 & 6.08 & -0.499 & 1.19 & 6.19 \\
\hline-0.75 & -0.748 & 2.00 & 10.3 & -0.748 & 1.74 & 10.5 \\
\hline-1.00 & -0.997 & 2.62 & 14.5 & -0.997 & 2.29 & 14.8 \\
\hline-1.25 & -1.25 & 3.17 & 18.8 & -1.25 & 2.81 & 19.1 \\
\hline-1.50 & -1.49 & 3.72 & 23.0 & -1.49 & 3.36 & 23.3 \\
\hline-1.75 & -1.74 & 4.24 & 27.3 & -1.74 & 3.89 & 27.6 \\
\hline-2.00 & -1.99 & 4.78 & 31.6 & -1.99 & 4.48 & 31.7 \\
\hline
\end{tabular}

Table 1: Mean longitudinal acceleration $\left(a_{x}\right)$, mean power loss $\left(P_{\text {loss }}\right)$ and mean regenerative power $\left(P_{\text {regen }}\right)$ of fixed ratio braking force distribution and optimal braking allocation schemes at different target longitudinal accelerations $\left(a_{x}^{\text {ref }}\right)$.

\section{Discussion}

The offline optimisation procedure presented in this paper enables significant savings in the motor power losses and thereby yields small increases in the regenerative power available for charging the battery during braking manoeuvres, compared to a simpler allocation method in which the frontto-total braking force ratio is constant at $65 \%$. For this specific type of motor, the method provides benefits in a small region of the torque demand and motor speed operating space. For other types of motor with a more pronounced variation in efficiency under different operating conditions, larger increases in regenerative power may be expected (see, for example, the motors used in [20]). Moreover, the benefits of the optimal braking scheme could be evident across a wider range of conditions. In addition to fully electric vehicles, the method could also be applied to hybrid cars in order to determine the distribution of regenerative braking between the front and rear wheel actuators. The constraints from the braking regulations are explicitly incorporated into the optimisation procedure and are thus embedded in the look-up tables; consequently, the wheel torques will satisfy the braking regulations and online adjustment with respect to the associated constraints is not required.

The method presented here may require adjustment to enable it to be applied on actual electric vehicles. Firstly, the equations corresponding to the friction constraints have been developed assuming a friction coefficient of one. In reality, different values of friction coefficient are likely to be encountered. As a result, for implementation of the method, it may be necessary to add the friction coefficient as a variable in the look-up tables so that the braking regulations could be met for different surface conditions. Alternatively, two different look-up tables could be used for high and low friction levels, with the latter condition being detected on the basis of wheel-lock behaviour. The constraints could also be evaluated online according to estimates of the friction coefficient. A simple online optimisation procedure (e.g. using quadratic programming) could be used to drive the braking distribution as close as possible to the values as determined by the look-up table within the constraints. In addition to the high level controller, the control allocation block will operate in conjunction with other control elements; for example, a wheel slip controller will be used in parallel to prevent situations of wheel lock. 
The power losses from the electric motors during regenerative braking are the target of the minimisation procedure presented in this paper. There are, however, other important sources of energy loss during the operation of a fully electric vehicle. For instance, the magnitude of power losses due to longitudinal tyre slip can be influenced by the control allocation scheme. Therefore, further developments will incorporate additional power loss sources into the cost function used for offline optimisation in order to minimise the overall power loss and thus maximise the regenerative power produced during operation.

The method demonstrated in this work may be further extended in order to allow more general manoeuvres incorporating cornering. In previous work, a corrective yaw moment has been applied in order to modify the understeer characteristic (the steady state relationship between the lateral acceleration and steering wheel angle [18]) and therefore the handling properties of the car [19]. The combined traction and yaw moment targets give (approximate) wheel torque totals for the leftand right-hand sides of the vehicle. Optimum wheel torque allocation may then be approached analogously to the method described in this paper, with separate look-up tables - with the leftor right-hand wheel torque demand and average motor speed as inputs - determining the frontto-total wheel torque ratio for the left- and right-hand wheels, $\gamma_{l}^{*}$ and $\gamma_{r}^{*}$. As an alternative, the yaw moment target could be added to the offline optimisation procedure and resulting look-up table, so that the method could be used in more general manoeuvres. The potential for power loss reductions for various types of manoeuvres involving cornering has been demonstrated in an offline study [20]. Future work will focus on expanding the method to allow it to be used online for more general manoeuvres which incorporate the generation of significant yaw moments. Finally, the control allocation method will shortly be validated for different types of duty cycle on a four-wheel drive, fully electric vehicle.

\section{Conclusions}

A method for maximising the regenerative power produced during braking of a fully electric vehicle has been developed which incorporates braking regulations constraints. The method does not rely on online optimisation and can therefore be implemented in real time onboard actual fully electric vehicles. The optimal braking force distribution approach yields power loss reductions and allows small increases in the total regenerative power compared with a simpler scheme in which the proportions of braking force delivered by the front and rear axles are fixed.

\section{Acknowledgment}

This research received funding from the European Union Seventh Framework Programme FP7/20072013 under grant agreement no. 284708. Thanks go to Michele Camporeale and Yiannis Matthaiou for their help with the simulations of the study.

\section{References}

[1] White House National Economic Council. Advanced Energy Initiative; 2006.

[2] Campbell CJ, Laherrère JH. The end of cheap oil. Scientic American. 1998.

[3] Hallock JL, Tharakan PJ, Hall CAS, Jefferson M, Wu W. Forecasting the limits to the availability and diversity of global conventional oil supply. Energy. 2004;29(11):1673-1696. 
[4] Sioshansi R, Denholm P. Emissions impacts and benefits of plug-in hybrid electric vehicles and vehicle-to-grid services. Environmental science \& technology. 2004;43(4):1199-1204.

[5] Eberle U, von Helmolt R. Sustainable transportation based on electric vehicle concepts: a brief overview. Energy \& Environmental Science. 2010;3(6):689-699.

[6] Maggetto G, Van Mierlo J. Electric and electric hybrid vehicle technology: a survey. In: IEEE Seminar on Electric, Hybrid and Fuel Cell Vehicles; 2000. p. 1-11.

[7] Affanni A, Bellini A, Franceschini G, Guglielmi P, Tassoni C. Battery choice and management for new-generation electric vehicles. IEEE Transactions on Industrial Electronics. 2005;52(5):1343-1349.

[8] Tate ED, Harpster MO, Savagian PJ. The electrification of the automobile: from conventional hybrid, to plug-in hybrids, to extended-range electric vehicles. SAE Paper 2008-01-0458. 2008.

[9] Savaresi SM, Tanelli M. Active braking control systems design for vehicles. Springer; 2010.

[10] de Castro R, Araújo RE, Tanelli M, Savaresi SM, Freitas D. Constrained quadratic programming techniques for control allocation. Vehicle System Dynamics. 2012;50:71-94.

[11] Chuanwei Z, Zhifeng B, Binggang C, Jingcheng L. Study on regenerative braking of electric vehicle. In: IPEMC 2004. The $4^{\text {th }}$ International Power Electronics and Motion Control Conference; 2004. p. 836-839.

[12] Härkegård O, Glad ST. Resolving actuator redundancyoptimal control vs. control allocation. Automatica. 2005;41(1):137-144.

[13] Chen Y, Wang J. Energy-efficient control allocation with applications on planar motion control of electric ground vehicles. In: American Control Conference (ACC); 2011. p. 2719-2724.

[14] Chen Y, Wang J. Fast and global optimal energy-efficient control allocation with applications to over-actuated electric ground vehicles. IEEE Transactions on Control Systems Technology. 2012;20(5):1202-1211.

[15] Economic Commission for Europe. Uniform Provisions concerning the approval of vehicles of categories M, N and O with regard to braking. Addendum 12: Regulation No. 13. 2008.

[16] Chu L, Sun W, Yao L, Zhang Y, Ou Y, Wei W, et al. Integrative control strategy of regenerative and hydraulic braking for hybrid electric car. In: IEEE Vehicle Power and Propulsion Conference, 2009 (VPPC'09); 2009. p. 1091-1098.

[17] Yuan X, Wang J, Colombage K. Torque distribution strategy for a front and rear wheel driven electric vehicle. In: 6th IET International Conference on Power Electronics, Machines and Drives (PEMD 2012). Bristol, United Kingdom; 2012.

[18] Gillespie TD. In: Fundamentals of Vehicle Dynamics. SAE; 1992.

[19] De Novellis L, Sorniotti A, Gruber P. Optimal wheel torque distribution for a four-wheel-drive fully electric vehicle. SAE International Journal of Passenger Cars. 2013;6(1):128-136.

[20] Pennycott A, De Novellis L, Gruber P, Sorniotti A. Enhancing the energy efficiency of fully electric vehicles via the minimization of motor power losses. In: IEEE International Conference on Systems, Man, and Cybernetics. Manchester, U.K.; 2013. 